\title{
Erratum: Structural Analysis of ZnO Nanowires Synthesized by Using a Low-temperature Hydro-thermal Method
}

\author{
[J. Korean Phys. Soc. 60, 1794 (2012)] \\ SangHyo Lee, JunSeok LeE, WonBae Ko, TaeSeong KAng and JinPyo HonG* \\ Research Institute for Natural Science, Novel Functional Materials and Devices Laboratory, \\ Department of Physics, Hanyang University, Seoul 133-791, Korea \\ SeungNam CHA \\ Frontier Research Laboratory, Samsung Institute of Advanced Technology, Yongin 446-712, Korea
}

DOI: $10.3938 /$ jkps.61.169

The acknowledgement was submitted incorrectly. It is corrected from 'This work was supported by a grant from the Korea Research Foundation funded by the Korean government (MOEHRD: 2010-0014680)' to 'This work was supported by the research fund of Hanyang University (HY-2005-M)'.

*E-mail: jphong@hanyang.ac.kr 